\title{
Spectrum of Short-Period Core Phases in Relation to the Attenuation in the Mantle ${ }^{1}$
}

\author{
Hiroo Kanamori ${ }^{2}$ \\ Seismological Laboratory, California Institute of Technology, Pasadena
}

\begin{abstract}
Spectral analysis is made of the records of the short-period reflected core phases from array stations at Tonto Forest, Arizona. Three earthquakes having $\Delta=32.5^{\circ}, 49.0^{\circ}$, and $62.8^{\circ}$ are studied. Spectral ratios of $S c S$ to $S c P$ and $P c S$ to $P c P$ are calculated in order to estimate the differential effect of attenuation on $P$ and $S$ waves. The possible maximum value of average $Q$ for shear in the mantle, $\bar{Q}_{\beta}$, is about 324 for the period range 1.5 to $5 \mathrm{sec}$. Using the value of $\bar{Q}_{\alpha}$, average $Q$ for $P$ waves, that was previously estimated as 435 through the spectral analysis of $P c P$ phase $\bar{Q}_{\beta}$ and $\bar{Q}_{\alpha} / \bar{Q}_{\beta}$ can be estimated at 230 and 1.90, respectively.
\end{abstract}

Introduction. The attenuation of elastic waves within the mantle has been studied by means of a variety of methods. To relate the observed attenuation to its physical mechanism, it is necessary to determine the distribution of $Q$, the dimensionless quality factor, with depth, the frequency dependence of $Q$ and the relation between the $Q_{a}, Q$ for $P$ waves, and $Q_{\beta}, Q$ for $S$ waves.

In this paper the attenuation of short-period ( $T=1.5 \sim 5 \mathrm{sec}$ ) body waves is determined through the spectral analysis of core phases such as $P c P, P c S, S c P$, and $S c S$. Three components seismograms recorded by the JohnsonMatheson short-period instruments at the Tonto Forest Seismological Observatory, Arizona, are used for the analysis. For the period September 1963 to August 1964 three pairs of $S c S$ and $S c P$ phases and one pair of $P c P$ and $P c S$ phases were clearly recorded. Table 1 lists the earthquakes, phases, and components used for the analysis. Figure 1 shows the E-W and N-S components of $S c S$ and $Z$ components of $S c P$ phase of January 26, 1964, earthquake. The phase identifications were mainly based on the arrival time. Whenever available, the arrival times of the corresponding phases at other stations at Blue Mountain, Cumberland Plateau, Uinta Basin, and Wichita Mountains were referred to for confirmation.

1 Contribution 1418, Division of Geological Sciences, California Institute of Technology.

${ }^{2}$ On leave from Geophysical Institute, Tokyo University.
Using the spectral ratio of either $S c S$ to $S c P$ or $P c S$ to $P c P$ and introducing several reasonable assumptions, we can determine the differential effect of attenuation on $P$ and $S$ waves from which the possible range of $Q_{\beta}$ can be determined.

Analysis. The ray paths of $S c S$ and $S c P$ phases are schematically illustrated in Figure 2. We will write the frequency spectrum of $S C S$ and $S c P$ phases at $B$ as $S c S(f)$ and $S c P(f)$, respectively.

Then

$$
\begin{array}{r}
S c S(f)=k I(f) S(f) \\
\cdot \exp \left\{-\pi f \int_{c,} \frac{d s}{Q_{\beta} V_{\beta}}\right\} \\
S c P(f)=k^{\prime} I(f) S^{\prime}(f) \\
\cdot \exp \left\{-\pi f \int_{c_{\nu}} \frac{d s}{Q_{\alpha} V_{\alpha}}\right\}
\end{array}
$$

where

$I(f)$, instrument response.

$S(f), S$-wave spectrum at $P$.

$S(f), S$-wave spectrum at $P^{\prime}$.

$C_{S}$, ray path $P \rightarrow B$.

$C_{P}$, ray path $P^{\prime} \rightarrow B$.

$V_{a}, P$-wave velocity.

$V_{B}, S$-wave velocity.

$k$ represents the amplitude diminution of $S c S$ phase due to various causes, such as the reflection at the core boundary and the geometrical spreading of the ray; $k^{\prime}$ is the corresponding quantity for $S c P$ phase. The integrations are 


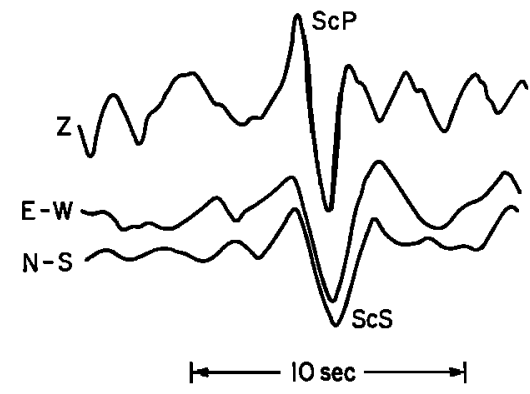

Fig. 1. Short-period seismograms recorded at Tonto Forest, Arizona, showing $Z$ component of $S c P$ phase and $\mathrm{E}-\mathrm{W}$ and N-S components of $S c S$ phase.

taken along $C_{s}$ and $C_{P}$ in (1) and (2), respectively. In the above, it is implicitly assumed that no spectral distortion is caused by the core boundary and the crustal layering. It has been inferred that the core boundary is well defined even for 1-sec period waves [Kanamori, 1967]. For the period range studied here, it is probably reasonable to regard it as a sharp boundary. The effect of the crustal layering can be estimated by Haskell's method [Haskell, 1962]. As will be discussed later, the crustal effect can be ignored in the present analysis.

We will further assume that

1. $S(f)=S^{\prime}(f)$.

2. $Q_{a}=\eta Q_{p} \quad(\eta$, constant $)$.

3.

$$
\int_{c,} \frac{d s}{Q_{\alpha} V_{\alpha}}=\frac{1}{1.82 \eta} \int_{c,} \frac{d s}{Q_{\beta} V_{\beta}}
$$

Assumption 1 is reasonable for two reasons. First, the difference of the take-off angle of

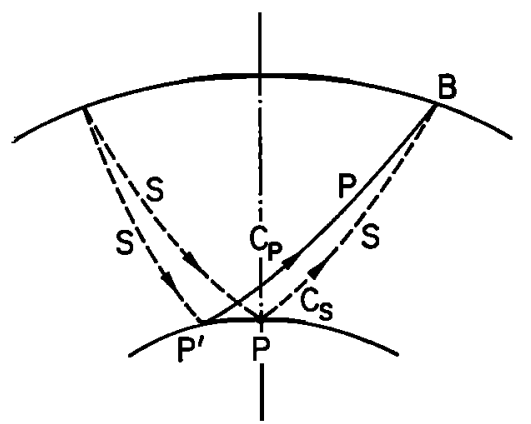

Fig. 2. Schematic illustration of ray paths of $S c S$ and $S c P$ phases. 
$S c P$ and $S c S$ at the source is smaller than $7^{\circ}$ even for the epicentral distance as large as $60^{\circ}$. Second, since the attenuation in the lower mantle is probably very small, the slight difference between the paths $S \rightarrow P^{\prime}$ and $S \rightarrow P$ does not greatly change the spectrum of $S$ waves. In assumption 3 , the replacement of the path is made for the same reasons, and the mean ratio 1.82 of the $P$-wave velocity to $S$-wave velocity within the mantle is introduced.

Dividing equation 1 by equation 2 and taking the logarithm of the absolute value, we have

$\log \left|\frac{S c S(f)}{S c P(f)}\right|=\log \left(\frac{k}{k^{\prime}}\right)-\pi \frac{T_{\beta}(\Delta) f}{\bar{Q}_{d}(\Delta)} \log e$

where

$$
\begin{aligned}
& \bar{Q}_{d}(\Delta)=\bar{Q}_{\beta}(\Delta) /(1-1 /(1.82 \eta)) \\
& \bar{Q}_{\beta}(\Delta)=T_{\beta}(\Delta) / \int_{c .} \frac{d s}{Q_{\beta} V_{\beta}} .
\end{aligned}
$$

$\Delta$ is the epicentral distance, and $T_{\beta}(\Delta)$ is the travel time of the $S$ wave from $P$ to $B$ and is equal to one-half of the travel time of $S c S$. $\bar{Q}_{\beta}(\Delta)$ is the average $Q_{\beta}$ along the path $C_{8}$. The average $Q_{\beta}$ usually defined corresponds to $\bar{Q}_{\beta}(0)$. The dependence of the ratio $\bar{Q}_{\beta}(\Delta) / \bar{Q}_{\beta}(0)$ on $\Delta$ is slight. $\bar{Q}_{\beta}(\Delta) / \bar{Q}_{\beta}(0)$ for CIT11 velocity model [Anderson and Toksöz, 1963] and MM8' $Q_{\beta}$ model, which represents a minor modification of the model given by Anderson et al. [1965], was calculated and tabulated in Table 2. Dependence of $\bar{Q}_{\beta}(\Delta) / \bar{Q}_{\beta}(0)$ on the distance will be about the same for other $Q$ models in which attenuation is less in the lower mantle than in the upper mantle.

Using (3) we can determine $\bar{Q}_{d}$ from the slope of logarithmic plot of the spectral ratio against

TABLE 2. Dependence of $\bar{Q}_{\beta}(\Delta)$ on $\Delta$

\begin{tabular}{cc}
\hline$\Delta, \operatorname{deg}$ & $\bar{Q}_{\beta}(\Delta) / \bar{Q}_{\beta}(0)$ \\
\hline 0 & 1.00 \\
10 & 1.00 \\
20 & 1.02 \\
30 & 1.04 \\
40 & 1.07 \\
50 & 1.11 \\
60 & 1.16 \\
70 & 1.21 \\
80 & 1.26 \\
\hline
\end{tabular}



Fig. 3. Amplitude spectrums of $S c S$ and $S c P$ phases for earthquake of November 3, 1963. Dots are the spectral ratios of $S c S / S c P$.

frequency. Since there are many uncertainties in the absolute amplitude originating from the reflection at the core boundary, local crustal structure, and the instrument magnification, the absolute amplitude ratio $k / k^{\prime}$ was not used, and only the relative spectral ratio was used.

The spectral analysis of the records was made in the following manner. The time record having $2 T_{m}$ duration was first taken using the time window

$$
\begin{aligned}
w(t) & =0.54+0.46 \cos \frac{\pi t}{T_{m}} & & |t| \leq T_{m} \\
& =0 & & |t|>T_{m}
\end{aligned}
$$

$w(t)$ was centered at the arrival time of the main pulse. Then it was digitized at every 0.2 sec. Two values for $T_{m}, 5$ and $10 \mathrm{sec}$, were tried. In case of $T_{\mathrm{n}}=10 \mathrm{sec}$ the spectrum was slightly jagged compared with that for $T_{\mathrm{m}}=5 \mathrm{sec}$, but the general trend was about the same.

In Figures 3, 4, and 5, the normalized spectrums for $T_{m}=5$ sec are shown. When two traces were available for one component, the average spectrum was used. The shift of the $S c P$ spectrum on the frequency scale with respect to $S c S$ is clearly observed for all cases. For the calculation of the spectral ratio, harmonics whose amplitude is less than $10 \%$ of the 




Fig. 4. Amplitude spectrums of $S c S$ and $S c P$ phases for earthquake of January 26, 1964. Dots are the spectral ratios of $S c S / S c P$.

amplitude of maximum harmonic were not used. The ratio thus calculated is plotted by dots. The linear decrease with increasing frequency is clearly seen, as expected. Straight lines are fitted to determine the slope of the spectral ratio.

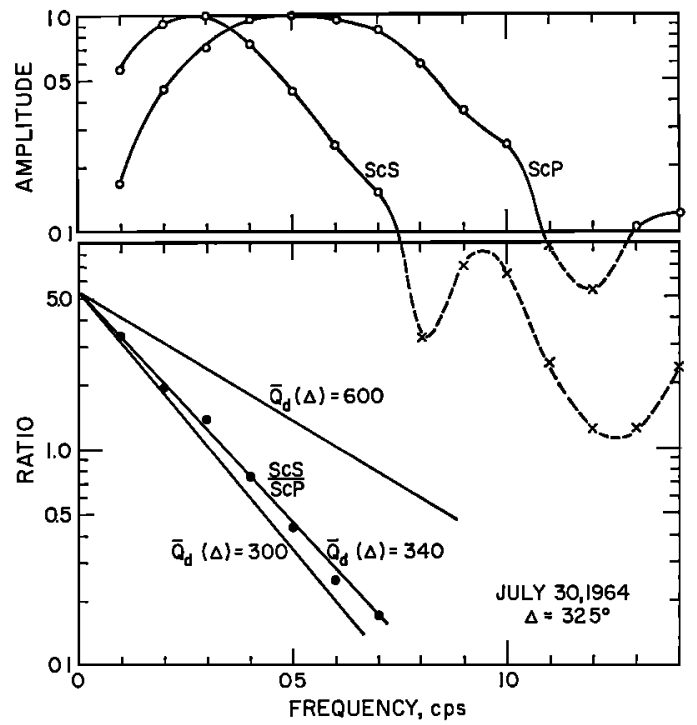

Fig. 5. Amplitude spectrums of $S c S$ and $S c P$ phases for earthquake of July 30, 1964. Dots are the spectral ratios of $S c S / S c P$.
TABLE 3. Summary of Results

\begin{tabular}{ccccc}
\hline $\begin{array}{c}\text { Earthquake } \\
\text { Number }\end{array}$ & $\begin{array}{c}\Delta, \\
\operatorname{deg}\end{array}$ & Phase & $\bar{Q}_{d}(\Delta)$ & $\bar{Q}_{d}(0)$ \\
\hline 1 & 49.0 & $S c S-S c P$ & 350 & 318 \\
2 & 62.8 & $S c S-S c P$ & 360 & 313 \\
3 & 32.5 & $S c S-S c P$ & 340 & 325 \\
& & $P c P-P c S$ & 350 & 340 \\
& & & Average & 324 \\
\hline
\end{tabular}

Two lines corresponding to $\bar{Q}_{d}(\Delta)=300$ and $\bar{Q}_{d}(\Delta)=600$ are drawn in the figure as references. The results together with the values corrected for the distance based on Table 2 are summarized in Table 3.

The same method applies to the $P c P$ and $P c S$ pair. The result is given in Figure 6 and Table 3.

The values of $\bar{Q}_{d}(0)$ determined from earthquakes having different distances and different phases are in fairly good agreement. The average value is 324 .

Discussion and conclusion. In order to estimate the crustal effect, which has so far been ignored, a crustal model as given by Table 4 was taken, and the transfer functions were calculated by the Haskell's method. As the angle of incidence of the up-going wave is about

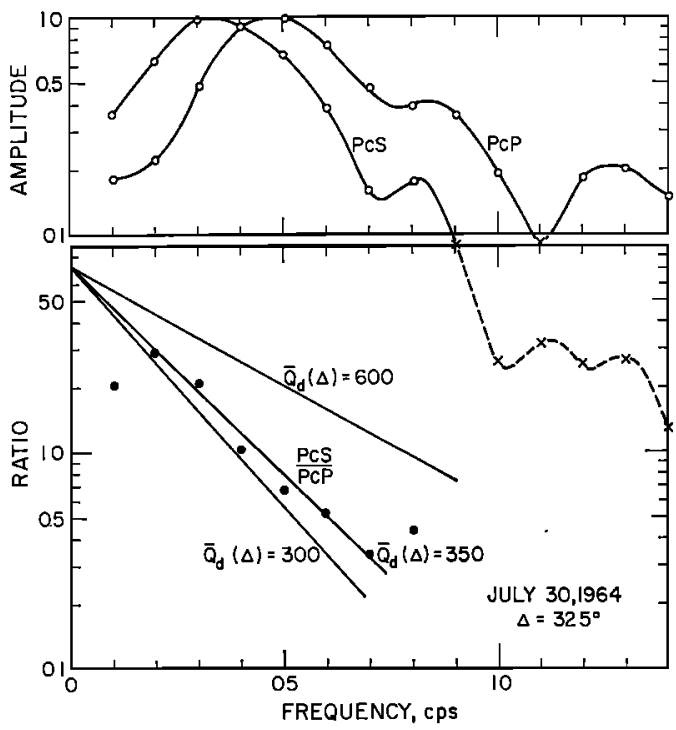

Fig. 6. Amplitude spectrums of $P c S$ and $P c P$ phases for earthquake of July 30, 1964. Dots are the spectral ratios of $P c S / P c P$. 
TABLE 4. Crustal Model Used in the Computetions of Transfer Functions

\begin{tabular}{|c|c|c|c|c|}
\hline & $\begin{array}{c}\text { Thickness, } \\
\text { km }\end{array}$ & $\begin{array}{c}P \text {-Wave } \\
\text { Velocity, } \\
\text { km/sec }\end{array}$ & $\begin{array}{c}\text { S-Wave } \\
\text { Velocity, } \\
\text { km/sec }\end{array}$ & $\begin{array}{c}\text { Density } \\
\mathbf{g} / \mathbf{c m}^{\mathbf{3}}\end{array}$ \\
\hline lot layer & 11.0 & 6.10 & 3.50 & 2.70 \\
\hline 2nd layer & 9.0 & 640 & 3.68 & 2.90 \\
\hline 3rd layer & 18.0 & 6.70 & 3.94 & 290 \\
\hline Half-space & & 8.15 & 4.75 & $\mathbf{3 . 3 0}$ \\
\hline
\end{tabular}

$10^{\circ} \sim 20^{\circ}$ at the base of the crust for the epicentral distance range concerned here, four cases are considered: $S V$-wave incident at the base of the crust with the angle $i_{\mathrm{s}}=10^{\circ}$ and $20^{\circ}$, and $P$-wave incident with the angle $i_{p}=$ $10^{\circ}$ and $20^{\circ}$. The results are given by solid curves in Figure 7. $\left|U_{s}\right|$ is the amplitude spectrum of the horizontal component of the surface displacement for the impulsive $S V$ input at the base of the crust. $\left|W_{p}\right|$ is the vertical component of the surface displacement for $P$-wave input. The spectrums are rather jagged, varying from 2 to 3 . In the present spectral analysis, however, time record of finite length was considered using the time window $w(t)$ given by (5). Let $H(f)$ represent the crustal transfer functions. The effective crustal transfer function $G(f)$ corresponding to the time record cut out by $w(t)$ centered at time $t_{1}$ can be written approximately as

$$
\begin{array}{r}
G(f)=e^{-2 \pi i s t_{1}} \int_{-\infty}^{+\infty} H(f) w\left(f^{\prime}-f^{\prime}\right) \\
\cdot e^{2 \pi i f^{\prime} t_{1}} d f^{\prime}
\end{array}
$$

where $W(f)$ is the Fourier transform of $w(t)$. The amplitude of $G(f)$ thus calculated for each case considered above is shown by the dotted curves in Figure 7. As expected from (6), the spectrum is smoothed and becomes essentially flat over the frequency range now considered. The crustal effect can, therefore, be ignored.

The value of $\bar{Q}_{d}$ (for brevity, $\bar{Q}_{d}(0), \bar{Q}_{\beta}(0)$, and $\vec{Q}_{\alpha}(0)$ are denoted by $\bar{Q}_{d}, \bar{Q}_{\beta}$, and $\bar{Q}_{\alpha}$ in the following), estimated at 324 , can be reduced to $\bar{Q}_{\beta}$ if we assume the value of $\eta$. Using (4), several solutions are given in Table 5 . It is obvious that $\bar{Q}_{d}$ gives possible maximum value of $\bar{Q}_{\beta}$. The value of $\eta$ depends on the physical mechanism of attenuation, and, though there is no widely accepted theory as to what value $\eta$ should take,

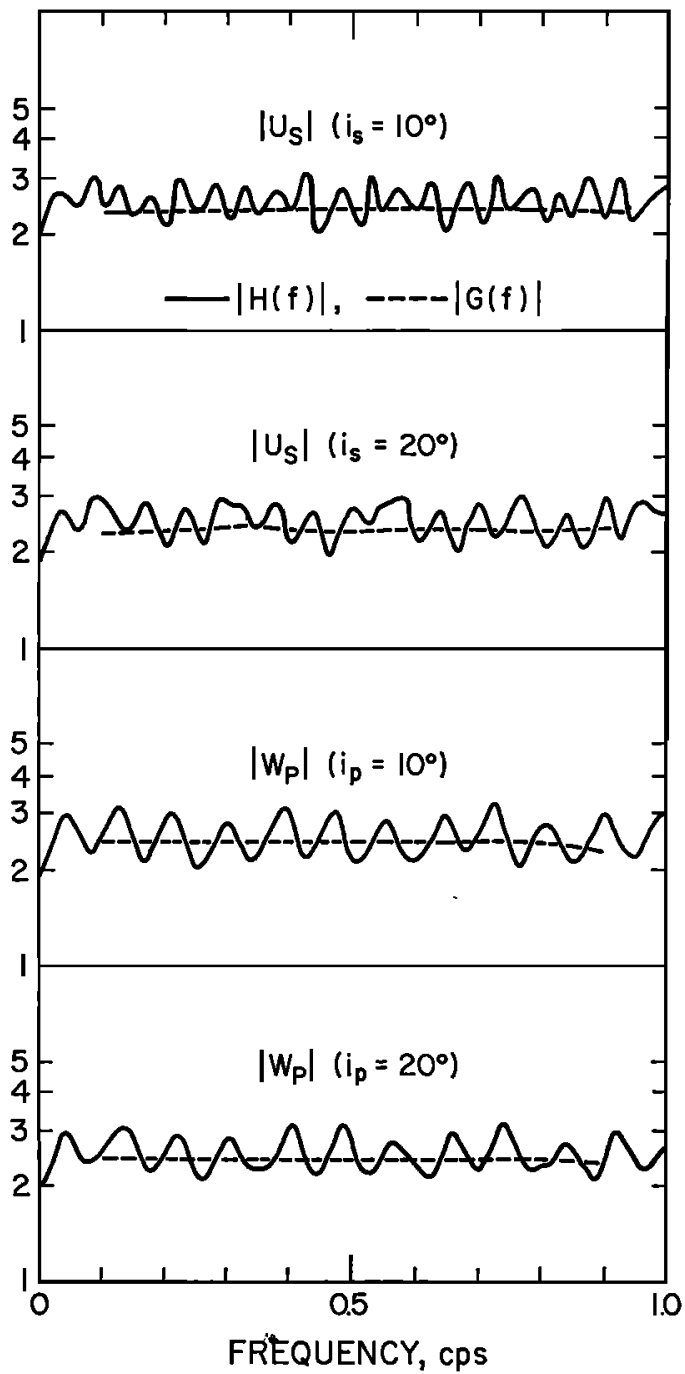

Fig. 7. Normalized crustal transfer functions for $S V$ incidence $\left(U_{*}\right)$ and $P$ incidence $\left(W_{p}\right)$. Solid curves are for time record of infinite length. Dotted curves are for time record of 10-sec length.

TABLE 5. Estimate of $\bar{Q}_{\beta}$ for Various $\eta$

\begin{tabular}{lrr}
\hline$\eta$ & $\bar{Q}_{\beta}$ & $\bar{Q}_{\alpha}$ \\
\hline 1 & 146 & 146 \\
$1.90^{*}$ & 230 & 435 \\
2 & 235 & 470 \\
3 & 264 & 792 \\
5 & 288 & 1440 \\
$\infty$ & 324 & $\infty$ \\
\hline
\end{tabular}

*Solution with $\bar{Q}_{d}=324$ and $\bar{Q}_{\alpha}=435$. 
$\eta$ of $1 \sim 3$ seems most probable. In this case the upper and lower bounds of $\bar{Q}_{\beta}$ are 150 and 260, respectively.

If we combine the value of $\bar{Q}_{\alpha}$ that was previously determined through the spectral analysis of short-period $P c P$ phase [Kanamori, 1967], we can determine $\eta$ and $\bar{Q}_{\beta}$ separately. It was shown that MM8' $Q$ distribution is a good approximation for $Q_{\alpha}$ around the period of $1 \sim 2$

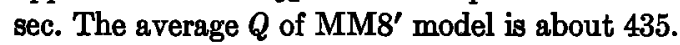
Using this value for $\bar{Q}_{\alpha}$ and the relation of (4) together with $\bar{Q}_{\alpha}=\eta \bar{Q}_{\beta}$, we obtain $\bar{Q}_{\alpha}=435$, $\bar{Q}_{\beta}=230$, and $\eta=1.90$, as shown in Table 5.

The value of $\bar{Q}_{\beta}$ obtained here appears to be definitely small compared with the previous estimates by Press [1956] $\left(\bar{Q}_{\beta}=500, T=11 \mathrm{sec}\right)$, Anderson and Kovach [1964] $\left(\bar{Q}_{\beta}=500, T=25\right.$ sec), and Kovach and Anderson [1964] $\left(\bar{Q}_{\beta}=600\right.$, $T=15 \sim 75 \mathrm{sec}$ ). Even allowing for the experimental uncertainties, this difference still seems significant. These results may indicate that $\bar{Q}_{\beta}$ decreases with increasing frequency. $\bar{Q}_{\beta}$ around 2 sec may be small by a factor of 2 compared with $\bar{Q}_{\beta}$ around $25 \sim 50$ sec. However, the short-period records are often disturbed by various reverberations of unknown origin. Since the effect of these reverberations on the individual spectrum is difficult to evaluate, it is necessary to increase the number of data before we can definitely establish the frequency dependence of $\boldsymbol{Q}$.
Acknowledgment. I am grateful to Drs. Don L. Anderson, C. B. Archambeau, J. N. Brune, and L. R. Johnson for various suggestions that are included in the final manuscript.

Research was partially sponsored by the Air Force Office of Scientific Research, Office of Aerospace Research, United States Air Force, under AFOSR contract AF-49(638)-1337.

\section{REFERENCES}

Anderson, Don L., Ari Ben-Menahem, and C. B. Archambeau, Attenuation of seismic energy in the upper mantle, J. Geophys. Res., 70, 14411448, 1965.

Anderson, D. L., and R. L. Kovach, Attenuation in the mantle and rigidity of the core from multiply reflected core phases, Proc. Natl. Acad. Sci. U. S. 51, 168-172, 1964.

Anderson, Don L., and M. Nafi Toksöz, Surface waves on a spherical earth, 1, Upper mantle structure from Love waves, J. Geophys. Res., 68, 3483-3500, 1963.

Haskell, Norman A., Crustal reflection of $P$ and $S V$ waves, J. Geophys. Res., 67, 4751-4767, 1962.

Kanamori, Hiroo, Spectrum of $P$ and $P c P$ in relation to the mantle-core boundary and attenuation in the mantle, J. Geophys. Res., 72, 559$571,1967$.

Kovach, R. L., and D. L. Anderson, Attenuation of shear waves in the upper and lower mantle, Bull. Seismol. Soc. Am., 54, 1855-1864, 1964.

Press, F., Rigidity of the earth's core, Science, $124,1204,1956$.

(Received September 22, 1966.) 